\title{
INSCRIPTIONS GRECQUES ET LES PEUPLES ITALIQUES, QUELQUES CAS D'ETUDE
}

Airton Pollini ${ }^{1}$

\section{Résumé}

L'article insiste sur les échanges entre Grecs et indigènes en GrandeGrèce, notamment lorsque ces derniers utilisent des modes d'expression empruntés du modèle grec, comme l'usage de l'alphabet, d'inscriptions gravées ou de monnaies. Ces emprunts peuvent être perçus à travers certains cas d'études, comme la borne de Tortora et les émissions monétaires des communautés italiques sous l'influence de Sybaris. Néanmoins, ces usages ne représentent pas forcément l'adoption des valeurs grecques, comme nous montre la réprobation du comportement homosexuel tel que l'ostrakon de Pisticci. Inversement, l'inscription sur l'olpè de Fratte indique la diffusion de la pratique pédérastique dans le milieu étrusque de la Campanie. Le témoignage épigraphique constitue ainsi un dossier fort adapté pour une analyse qui vise à confronter sources textuelles et artefacts, puisque ces inscriptions réunissent, sur un même objet, les approches d'analyse de la culture matérielle et textuelle, avec des analyses nuancées sur les modalités d'interaction culturelle entre Grecs et indigènes.

\section{Mots-clés}

Interaction culturelle; inscriptions; colonisation grecque; monde italique;homoérotisme.

\footnotetext{
1 Maître de Conférences, Université de Haute Alsace - Mulhouse, France. email:
} airton.pollini@uha.fr 


\section{Resumo}

O artigo enfatiza os intercâmbios entre gregos e nativos na Magna Grécia, especialmente quando os últimos utilizam formas de expressão emprestadas do modelo grego, como o uso do alfabeto, de inscrições gravadas ou de moedas. Estes empréstimos podem ser percebidos através de alguns estudos de caso, como o horos de Tortora e as emissões monetárias das comunidades itálicas sob a influência de Sybaris. No entanto, estes usos não representam necessariamente a adoção de valores gregos, como mostra a desaprovação do comportamento homossexual tal como o ostrakon de Pisticci. Por outro lado, a inscrição na olpè de Fratte indica a difusão da prática homossexual no ambiente etrusco da Campânia. A evidência epigráfica constitui assim um tipo de fonte bastante adequado para estudos cujo objetivo é de confrontar fontes textuais e artefatos, já que essas inscrições reúnem, num mesmo objeto, as abordagens de análise da cultural material e textual, com análises nuançadas sobre as modalidades de interação cultural entre os gregos e os nativos.

\section{Palavras-chave}

Interação cultural; inscrições; colonização grega; mundo itálico; homoerotismo. 
Pour l'étude de l'interaction entre textes et culture matérielle, l'approche relativement récente de l'archéologie historique fournit un cadre particulièrement pertinent (Orser Jr, 2000 ; Orser Jr, 2002. Voir aussi Meskell, 2001: 187-213 ; Lawrence e Shepherd, 2006: 71). Un développement approfondi sur son apport méthodologique est inutile ici, mais il est intéressant de rappeler ses enjeux majeurs. En effet, l'archéologie historique s'est fortement inspirée des problématiques de l'anthropologie et des sciences sociales et se concentrait, à l'origine, à l'étude de la société américaine formée après la conquête des Européens. Par la suite, les possibilités d'application de cette approche se sont élargies à tout contexte historique qui permet la confrontation entre sources écrites et culture matérielle (Funari, 1999; Funari, Zarankin, e Stovel, 2005 ; Funari, Oliveira, e Zarankin, 2010). Sans établir d'hiérarchie entre les deux types de sources (Small, 1995a: 4-5 ; Small, 1999: 122-136 ; Dyson, 1995: 25-44; Johnson, 1999: 23-36)1, son apport majeur est la méthode qui consiste à déconstruire les objets archéologiques selon une méthode littéraire d'interprétation de texte, analysant chaque détail séparément, pour ensuite reconstruire l'ensemble avec une compréhension approfondie. L'objectif qui se trouve en filigrane est celui de souligner des thématiques telles que l'exploitation des classes, les différences de statuts, l'histoire des genres. Par conséquent, l'accent est porté sur les relations de pouvoir entre groupes sociaux et individus, sur les mécanismes de domination et de résistance ${ }^{2}$. À ce propos, il est désormais admis que les vestiges archéologiques consentent d'appréhender les traces des couches sociales les plus basses, notamment par l'analyse attentive des objets du quotidien, dont notamment la céramique ${ }^{3}$. Dans notre cas précis, il s'agit également d'appréhender les rapports d'interaction interethnique, entre Grecs et populations italiques.

Notre point de départ est constitué d'un document épigraphique relevant d'une certaine volonté de délimitation d'un espace. Si l'idée même d'une pierre inscrite qui porte la mention d'une limite paraît évidente, dans le détail, l'analyse est bien plus complexe. Ainsi, avant de traiter spécifiquement de notre cippe, il est opportun d'afficher explicitement les

\footnotetext{
${ }^{1}$ Pour l'archéologie du monde grec classique, voir des exemples concrets : Ober, 1995: 91-123 ; Small, 1995b: 143-174.

2 Remarquons la possibilité d'utiliser des approches telles que les "Third wave feminists ", Meskell, 2001: 192-194, ou d'histoire du genre, Scott, 1986: 1053-1075.

${ }^{3}$ Voir commentaire sur la possibilité d'utilisation des méthodes de l'archéologie historique dans d'autres périodes historiques : Lawrence e Shepherd, 2006: 75 ; et particulièrement pour cette transposition vers les mouvements coloniaux des Grecs, Phéniciens et Romains : Cunliffe, 2006: 317.
} 
écueils auxquels nous sommes confrontés lors de l'étude de ce type de document.

À ce propos, J. Ober (Ober, 1995: 91-123) ${ }^{4}$ plaide pour la plus grande prudence dans l'interprétation des signes matériels des limites, qui doivent être systématiquement contextualisés. À ce titre, l'exemple le plus connu est celui des deux bornes de l'agora d'Athènes, découvertes en 1938 (I 5510) et 1967 (I 7039) et retrouvées in situ, dont la première immédiatement à l'est de la Tholos, à l'endroit où la voie ouest de l'agora se bifurque. La pierre porte, sur les côtés supérieur et oriental, un court

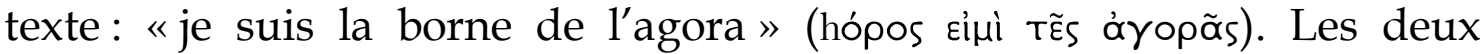
inscriptions sont datées vers 500 av. J.-C. (Shear, 1939: 205-206; Thompson, 1968: 61-63; commentaires dans Lalonde, Langdon, e Walbank, 1991, H. 25 et H. 26, p. 27, pl. 2) 5 .

La fonction de démarcation de l'agora semble bien claire, ce qui était important à plusieurs titres. Tout d'abord, cela servait à délimiter le domaine de l'espace public de la place centrale et empêchait toute appropriation privée. Ensuite, ces bornes servaient notamment à indiquer la limite à ne pas franchir par plusieurs catégories de personnes dont l'accès était interdit à l'agora, en particulier des meurtriers ou ceux frappés d'atimie, c'est-à-dire la privation des droits de citoyen 6 . L'agora étant la place publique consacrée aux activités politiques et économiques, cette interdiction d'accès avait des conséquences non seulement pour un citoyen qui ne pouvait plus participer aux décisions de la communauté, mais aussi à d'autres catégories sociales, étrangers notamment, empêchées d'acheter ou de vendre au marché. En revanche, dès la fin du Ve siècle, la première borne (I 5510) était déjà enterrée et ne servait plus à délimiter l'espace de l'agora, tandis que la deuxième (I 7039) a été recouverte dans le IIIe siècle av. J.-C.

Premièrement, dans le cas des bornes de l'agora, pour que l'énoncé soit fonctionnel, la pierre doit rester à l'emplacement même des limites qu' elle indique et le lecteur doit pouvoir identifier ce que représente l'espace

\footnotetext{
${ }^{4}$ En particulier p. 96 : « Looking at how several texts treat horoi, and examining the horos as an 'artifactual text' - as a text that is an artifact, and at the same time an artifact that is a text should help to elucidate some methodological problems involved with moving back and forth between texts and artifacts, history and archaeology. "

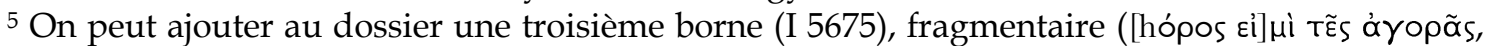
[hóros ei]mí tês agorâs), mais qui doit restituer le même texte et la même datation, Shear, 1940: 266 et Lalonde, Langdon e Walbank, 1991, H. 27, p. 27. Sur ces bornes, voir les commentaires de F. Longo dans Greco, Longo, Di Cesare e Marchiandi, 2014: 1096-1097.

${ }^{6}$ Selon l'interprétation de Hansen, 1976: 55-62, plusieurs passages indiquent les caractéristiques des sentences d'atimie comme l'interdiction d'accès à l'agora, entendue comme la place du marché, et aux sanctuaires (Lysias, VI Contre Andocide, 9 et 24). Voir aussi Démosthène, XXII, 77 ; XXIV, 60 ; Eschine, I, 164 ; II, 148 ; III, 176.
} 
qu'elle délimite, en l'occurrence, l'agora. De plus, ce document véhicule aussi un ordre, du moins une mise en garde: le passant doit être conscient des conséquences qu'il encoure s'il dépasse cette limite, comme dans le cas de quelqu'un qui n'aurait pas le droit d'accéder à l'agora. Ici, le message est implicite, mais reste assez clair. Il est relativement aisé d'identifier l'espace délimité par la borne; on sait que l'autorité qui définit les limites est la cité d'Athènes, ou plus précisément la communauté des citoyens athéniens, le démos; enfin, on peut supposer une large connaissance des conditions d'accès à l'agora et les peines pour ceux qui les transgressent. À partir de la confrontation avec le témoignage littéraire à notre disposition, le chercheur contemporain est donc capable de saisir la plupart des enjeux portés par ces bornes. Les mêmes conditions interviennent pour les bornes de délimitation de sanctuaires : les conditions d'accès, les rituels prévus à l'entrée et les peines pour les transgressions devaient être connus par une très large portion de la population.

Dans d'autres cas, en revanche, les nombreuses bornes portant simplement la mention horos constituent un dossier beaucoup plus complexe. Ici, même si les pierres étaient restées en place, la majorité des informations sont implicites et insaisissables par le chercheur contemporain: ce qui est délimité, l'autorité responsable, les règles d'accès à cet espace, quel qu'il soit, et les éventuelles sanctions lors de leur infraction. Dans le cas de ces bornes assez laconiques, il peut s'agir de domaines publics, sacrés ou privés, donc des délimitations relatives à des espaces accessibles à la majorité ou bien des propriétés des particuliers. Rien n'empêche d'ailleurs qu'une borne portant l'inscription horos soit déplacée et, avec le même texte, l'espace qu'elle délimite puisse changer. Cet exemple montre clairement que, même dans le cas d'une mention aussi directive et soi-disant simple, le message qu'elle véhicule dépend d'un contexte bien plus complexe qu'uniquement sa datation et son emplacement. Enfin, des bornes anépigraphiques (sans aucune inscription) pouvaient aussi délimiter des espaces. Dans ce dernier cas, toutes les informations sont perdues pour nous puisqu'il est pratiquement impossible de reconnaître les fonctions d'une pierre sans aucune marque particulière, même si sa forme peut éventuellement faire penser à des bornes.

\section{Inscription indigène de Tortora}

Ces remarques introductives ont pour objectif $\mathrm{d}$ 'insister sur les difficultés d'interprétation de documents épigraphiques qui sembleraient, a priori, porter un message évident. Dans le cas des objets inscrits émanant de 
communautés non-grecques et en contact avec les colons grecs d'Italie du Sud, ces précautions trouvent leur application renforcée.

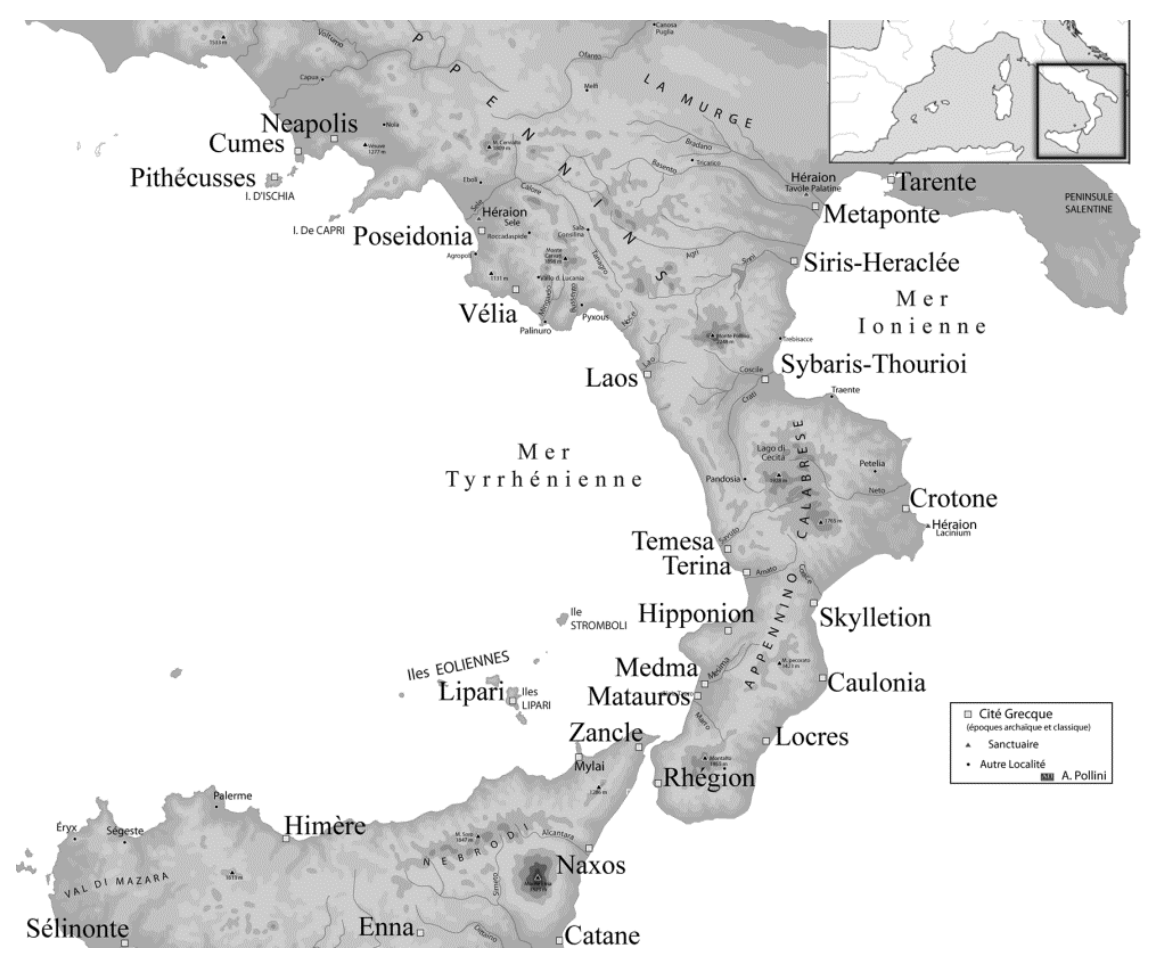

Carte de la Grande-Grèce et de la Sicile, avec l'indication des cités grecques coloniales. (C) Airton Pollini.

En ce qui concerne le dossier épigraphique en Grande-Grèce, le témoignage à notre disposition est très limité. En revanche, un cippe, découvert en 1991 à Tortora, sur la vallée du Noce et près de l'ancienne Laos, mérite notre attention ${ }^{7}$. La pierre calcaire rectangulaire fragmentaire, d'environ $100 \mathrm{~kg}$, est haute de $67 \mathrm{~cm}$, large de $37 \mathrm{~cm}$ et profonde de $21 \mathrm{~cm}$ et porte un texte, écrit en alphabet grec achéen, distribué sur les quatre faces latérales, avec cinq lignes sur les faces plus larges (A et C) et trois lignes sur les côtés courts (B et D), dont une surface illisible (D), ainsi que deux lignes sur la face supérieure $(E)^{8}$. Le texte est

\footnotetext{
${ }^{7}$ Nos remarques se fondent sur l'édition critique de l'inscription dans Lazzarini e Poccetti, 2001. Pour le contexte de la découverte, voir La Torre, 1995.

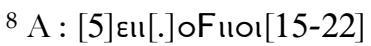

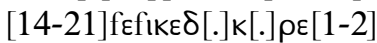

$[1-2] ı \sigma \theta \mu \alpha \delta \propto \mu \alpha \mathrm{T} \varepsilon \sigma \varepsilon[15-18]$

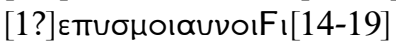

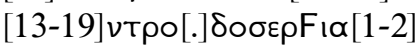

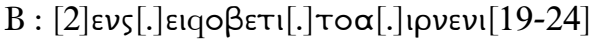

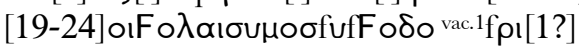

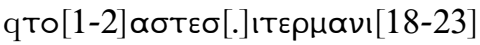


rédigé en lignes verticales, en écriture boustrophédon, et a été daté de la fin du VIe siècle av. J.-C. L'objet a été trouvé dans la localité de San Brancato, à Tortora, à environ 150 m d'une nécropole indigène, mais il ne semble pas avoir de rapport direct avec cette dernière. Les dimensions de la pierre plaident pour un emplacement d'origine certainement à proximité de la zone de sa découverte, sur une petite hauteur du plateau de San Brancato. Les prospections de surface n'ont livré aucune structure, mais le matériel archéologique permet d'affirmer l'existence d'un habitat archaïque situé entre la nécropole et le lieu de découverte de l'inscription.

Bien que la restitution du texte soit très complexe, quelques remarques sont néanmoins possibles. Premièrement, les populations locales ont utilisé l'alphabet grec sans modifications substantielles pour écrire une langue italique qui s'apparente à la même racine de ce qui devient l'osque plus tard. D'une part, l'interaction culturelle entre Grecs et autochtones est perceptible sous l'aspect de l'utilisation de l'alphabet dès l'époque archaïque, d'autre part, la conquête samnite de la fin du Ve siècle peut, sous l'aspect linguistique, être nuancée et des intenses rapports culturels entre les populations du centre et du sud de la péninsule italienne peuvent être identifiés dès au moins un siècle plus tôt. Deuxièmement, pour ce qui est du contenu, sans que l'on comprenne l'ensemble du texte, fragmentaire, quelques lemmes ont été identifiés. C'est la ligne 3 de la face B qui nous intéresse. L'usage de l'impératif en -tōd (restitué de qto[12]) serait caractéristique, en latin et dans les langues italiques, des dispositions normatives de textes législatifs publics ou de prescriptions dans un cadre religieux, où aucun lien n'est établi avec une institution

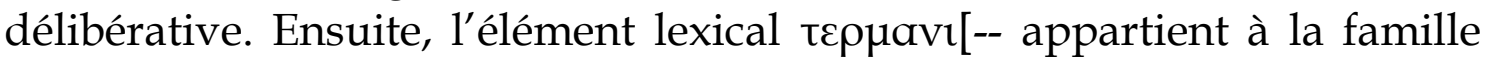

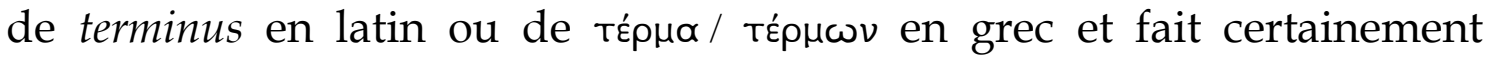
référence à la pierre elle-même en tant que borne, notamment par la corrélation avec le déictique $\varepsilon \sigma \varepsilon \imath$ (par segmentation d' $\mathrm{d} \sigma \tau \sigma[\varepsilon] \mathrm{l}$ ) qui accentuerait la fonction d'autoréférence du cippe. Enfin, dans la ligne 1 de la face $C$, sur le plan lexical, toFtı peut être relié au terme toutā-, qui se réfère à l'organisation ethnique et politique des communautés italiques ${ }^{9}$. Ici, les éditeurs ont restitué un composé prépositionnel qui pourrait se lier à toutā- pour indiquer «à l'intérieur, à l'extérieur, autour, devant, derrière ou près de la toutā- »(Lazzarini e Poccetti, 2001: 159),

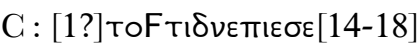

$[15-18] \sigma[.] \varepsilon \delta F \circ \lambda \circ \sigma f u f u F \circ \delta$ vac.3

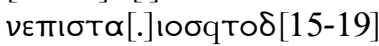

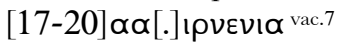

$\mathrm{E}:[4-5] v i \propto[.] \varepsilon[.] \Perp[?] \mathrm{U}$

[.] $\alpha \mu[$.$] vouF [6-9]$.

${ }^{9}$ Sur le terme toutā-/ touto et ses rapports avec les définitions des communautés d'Italie centrale, voir Bourdin, 2012: 240-266. 
c'est-à-dire du centre habité de la communauté. Ici, il y a probablement un sens identitaire fort de l'affirmation du groupe social, en tant qu'entité autonome.

Si le message complet du texte est pour le moment trop obscur, il est intéressant de souligner l'existence d'une borne inscrite faisait certainement état d'une limite relative à la communauté locale qui exprime son autonomie. Il est impossible d'affirmer précisément le type d'espace qui est délimité, mais il est plausible de soutenir qu'il s'agit d'un domaine soit public, soit sacré, en tout cas les éléments restitués écartent la possibilité d'un espace privé ou funéraire. L'identification d'un impératif corrobore l'interprétation d'un cippe qui expose, sur un lieu public ou sacré, un contenu de dispositions normatives par rapport à un espace délimité.

Tout commentaire supplémentaire sur ces questions serait totalement hasardeux. Toutefois, il s'agit d'un document exceptionnel qui montre une volonté de définition politique et identitaire d'un espace public ou sacré d'une communauté indigène à la fin du VIe siècle av. J.-C. et qui utilise, à cet effet, des moyens sans doute empruntés des pratiques grecques: l'usage de pierres inscrites et l'utilisation de l'alphabet grec, ainsi que l'idée même de démarcation d'espaces collectifs.

\section{Monnaies et alliance}

Dans l'optique de l'analyse de l'interaction culturelle entre Grecs et indigènes, un dossier numismatique mérite d'être évoqué. Il s'agit d'un certain nombre de monnaies qui portent le symbole de la cité grecque de Sybaris, le taureau, mais avec des ethniques qui font référence à d'autres communautés: Sirinos et Pyxoes; Ami ou Asi; Lainos ; So... ; et Pal/Mol. L'interprétation du témoignage numismatique est difficile et on a tenté d'identifier les lieux rapportés par ces monnaies comme des allusions soit à d'autres cités grecques sous l'influence sybarite, comme Siris ou Laos, soit à des centres indigènes, comme Pyxunte, Aminaia (Francavilla Marittima ?), Sontini10, Palinuro et Molpa (Greco e Gasparri, 1995: 73-74 ; Greco, 1992a: 89-93; De Juliis, 1996: 180 ; Parise, 2001; en dernier, Horsnaes, 2011). Si l'identification des ethniques est malaisée, aussi la chronologie de ces émissions n'est pas certaine et deux phases ont été proposées : une première série pour la légende Sirinos-Pyx dans le dernier tiers du VIe siècle, et une deuxième série pour les légendes Sirinos-Pyxoes, Ami, So... et Pal/Mol autour de 510 av. J.-C. (Taliercio Mensitieri, 2001: 128). Quels que soient les lieux indiqués par ces monnaies, la

\footnotetext{
${ }^{10}$ Sontini est cité par Pline l'Ancien, N.H., III, 15 (11), 98.
} 
numismatique corrobore la notion d'une sphère d'influence de la cité grecque de Sybaris assez étendue, comprenant plusieurs sites entretenant des contacts importants avec la cité achéenne. Sur ce point, la frappe de monnaie et l'adoption d'une iconographie émanant de Sybaris peuvent être vues comme un signe d'une forte interaction entre la cité grecque coloniale et plusieurs centres indigènes autonomes. Malgré toute la prudence qu'exige cette documentation numismatique, elle constitue la principale source matérielle pour l'affirmation de l'« empire» sybarite (Greco, 1992b: 463-465), dans le sens d'une organisation qui pourrait être comparable au modèle des hégémonies orientales "par districts », ce qui implique certes des contacts et une certaine forme d'hiérarchie dans les rapports, mais aussi une grande autonomie des centres indigènes (Bugno, 1999: 7-35, en particulier p. 26) ${ }^{11}$.

Dans ce sens, le dossier doit être complété par l'inscription trouvée dans le sanctuaire d'Olympie portant un accord entre Sybaris et les Serdaioi, avec la garantie de Poseidonia ${ }^{12}$. Si la lecture de cette inscription semble simple, son interprétation ne l'est point. En effet, des discussions se sont développées d'abord autour des possibles identifications de ces Serdaioi et des rapports avec l' « empire » de Sybaris, puis de la datation du traité (Greco, 1990)13. Suivant la majorité des commentateurs, le plus plausible est de situer cet ethnos du côté tyrrhénien, certainement au sud de Vélia, peut-être à proximité de Laos. En ce qui concerne la chronologie, rien ne nous permet de trancher définitivement la question si le traité fut établi par les Sybarites avant la destruction de leur cité par la cité voisine Crotone en 511 av. J.-C. ou bien par les réfugiés qui maintenaient leur dénomination ${ }^{14}$. Cette datation mènerait à penser que l'accord avec les

${ }^{11}$ La source principale est le passage de Strabon $(\mathrm{VI}, 1,13)$ à propos de la puissance de la cité de

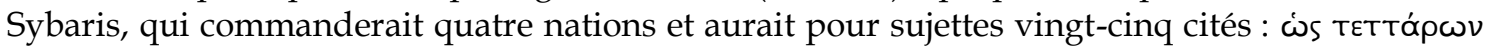

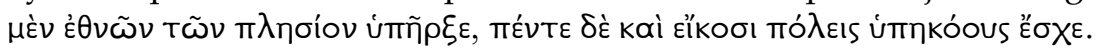

12 áppó $\chi \theta \varepsilon v$ oi $\Sigma u \beta \alpha \rho \tilde{-}-$

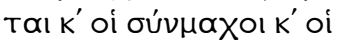

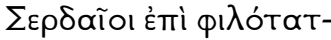

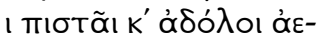

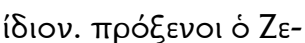

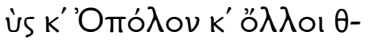

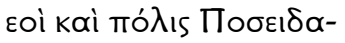

vía.

SEG 22, 1967, n. 336, musée d'Olympie, B. 4750. « Se sont unis les Sybarites et leurs alliés et les Serdéens [Serdaioi], en amitié fidèle et confiante, pour toujours. Sont témoins Zeus, Apollon, les autres dieux et la cité de Poseidonia » (trad. Bertrand). Cf. Van Effenterre e Ruzé, 1994, n. 42, p. 174-177 ; Dubois, 2002, n. 12, p. 36-40 ; Bertrand, 2004, n. 8, p. 36-37 et Nouveau choix 2005, n. 16, p. 95-97. Voir interprétation du vocabulaire de l'inscription dans Giangiulio, 1992 et Bugno, 2001: 322-325.

${ }^{13}$ Voir un bilan des discussions dans Polosa, 2000. Plus récemment, voir Lombardo, 2008 et Greco, 2013.

${ }_{14} \mathrm{M}$. Lombardo souligne l'analyse paléographique des formes des lettres qui plaide pour une fourchette comprise entre la dernière décennie du vie et le début du ve siècle av. J.-C., puis des 
Serdaioi était lié à l'installation des Sybarites à Laos et à Skidros, possiblement sur un territoire octroyé par cette communauté italique.

Le dossier est ensuite enrichi par dix-sept monnaies (MERD- $\Sigma$ EP $\triangle$ ) attribuées à une émission des Serdaioi, dont l'analyse stylistique les rapproche des monnaies de Poseidonia, malgré les différents étalons. La datation proposée entre dans une fourchette comprise entre 510 et 490 av. J.-C. (Brousseau, 2010) ${ }^{15}$ et contribue, par conséquent, à l'hypothèse de la chronologie basse pour le traité. En tout cas, nous suivons l'observation de M. Lombardo sur la nécessité d'analyser les documents dans la chronologie et de forme dynamique (Lombardo, 2008: 51).

Dans tous les cas et indépendamment des divergences dans l'exégèse des sources, le dossier indique clairement des formes d'interaction culturelle forte entre colons grecs et communautés indigènes. Les documents qui relèvent du dossier sur $\mathrm{l}^{\prime}$ " empire» de Sybaris permettent de voir l'utilisation des codes d'affirmation identitaire, comme l'émission monétaire et l'apposition d'ethnique, en alphabet grec de surcroît, selon un modèle grec mais utilisés par des communautés indigènes.

\section{Une condamnation de la pédérastie à Pisticci}

Dans l'arrière-pays de la cité grecque de Métaponte, l'analyse fine de la culture matérielle provenant du site de Pisticci (Osanna, 1992: 83-84, n. 18 ; Barberis, 1999: 75, n. A40 ; Castoldi, 2007 ; Bottini e Lecce, 2013: 50$54)^{16}$ permet également des commentaires sur l'interaction entre Grecs et indigènes en Italie méridionale.

Le centre indigène de Pisticci montre des signes d'une présence importante et continue depuis l'âge du fer et pendant tout le VIe siècle, sans intervention directe des Grecs. L'établissement suit un modèle polycentrique, connu par ailleurs dans l'arrière-pays italique, avec au moins trois secteurs d'habitat et leur nécropole associée. En effet, une zone d'habitat à S. Maria del Casale comporte une nécropole, avec plusieurs noyaux de tombes repartis dans toute la fourchette chronologique d'occupation du site. Dans le lieu-dit S. Leonardo en particulier, le mobilier des tombes se compose des céramiques indigènes, des fragments de lance en bronze et d'épée en fer dans les tombes masculines ainsi que des riches parures en bronze pour les sépultures

\footnotetext{
raisonnements historiques, pour proposer une datation après la défaite de 511/510 av. J.-C. Lombardo, 2008: 55. Voir aussi Dubois, 2002: 36-38.

${ }^{15}$ À partir des aspects stylistiques et à cause de la technique du double relief.

${ }^{16}$ Le territoire compris entre Pisticci et Ferrandina fait l'objet des recherches de l'université de Milan, sous la direction de M. Castoldi.
} 
féminines. Entre le VIIe et le VIe siècle, la céramique indigène, à décoration géométrique et sans décoration, ainsi que de la céramique de production grecque coloniale, côtoie un nombre important de vases grecs d'importation, notamment dans les secteurs de Via Di Giulio et de Matino Soprano. Dans la deuxième moitié du VIe siècle, une plus forte influence grecque se vérifie et, sur les pentes de la colline, ont été trouvés de nombreux fragments de vases à figures noires et rouges, ainsi que des objets en bronze. Enfin, un grand nombre de tombes situées dans diverses zones du centre moderne de Pisticci couvrent une fourchette chronologique large, allant du Ve au IVe siècle av. J.-C. Dans le tournant $\mathrm{du}$ siècle, les vases grecs d'importation, attiques notamment, sont remplacés par des vases de production coloniale locale ${ }^{17}$, y compris notamment des objets d'origine apulienne.

La présence indigène à Pisticci est suffisamment importante pour que M. Osanna (Osanna, 1992: 54) soutienne l'impossibilité d'une occupation grecque non seulement sur ce site, mais aussi dans toute la zone environnante. Ainsi, une hypothèse ancienne qui identifiait ici un phrourion est désormais totalement abandonnée.

Cette hypothèse d'une fortification se fondait sur la découverte d'un ostrakon, où l'on lit katámuy[ov] (infâme, débauché), en alphabet achéen, interprété comme une allusion à la pédérastie. Il est important de souligner que l'existence d'un objet inscrit en grec et sans doute portant une mention à la pratique de la pédérastie ne doit pas mener directement à l'identification d'une présence d'un noyau de population grecque, a fortiori d'un groupe de péripoloi dans une fortification (Tagliente e Lombardo, 1985 ; Bottini e Lecce, 2013: 54).

En effet, les interactions culturelles entre Grecs et indigènes peuvent relever de diverses formes. L'élément le plus intéressant ici est la condamnation d'une certaine pratique assez courante dans le monde grec mais faite selon les codes d'expression d'un modèle grec. L'usage d'un ostrakon, de l'alphabet et même de la langue grecque n'implique pas l'adoption d'une certaine pratique. Ainsi, on voit l'utilisation des codes empruntés d'une culture pour condamner une pratique qui y est souvent associée.

\footnotetext{
17 À ce propos, il est intéressant de rappeler que le premier peintre connu de la région de Métaponte est le peintre de Pisticci, indépendamment de l'impossibilité de déterminer avec précision son origine, cf. Denoyelle, 1997. Voir aussi Denoyelle e Iozzo, 2009.
} 


\section{Homoérotisme entre Grecs et indigènes à Fratte}

Dans une interprétation inverse à celle de l'ostrakon de Pisticci, c'est-àdire le témoignage d'une pratique pédérastique par des personnes de différentes origines ethniques, une inscription provenant d'une tombe de Fratte peut mener à une analyse plus nuancée.

En effet, les tombes du site de Fratte (Greco e Pontrandolfo, 1990) représentent l'un des meilleurs témoignages de l'emprise de Poseidonia sur la Campanie. Cette influence grecque est visible non seulement dans les échanges commerciaux, mais aussi dans contacts d'ordre culturel, voire privé. La découverte en 1963 d'une inscription en alphabet achéen sur une petite olpè datée du premier quart du Ve siècle dans la tombe vingt-six est très significative. L'étude attentive du vase lui-même a permis d'identifier son lieu de production comme étant la cité grecque voisine de Poseidonia et il a été gravé avant cuisson, dans un atelier poseidoniate. En plus de la simple présence de matériel céramique grec dans les tombes de Fratte, cette inscription nous renseigne aussi sur des rapports personnels entre les gens de diverses ethnies.

Le texte, en alphabet achéen, comparable avec les inscriptions dialectales de Poseidonia (Arena, 1996 ; Dubois, 2002), est le suivant :

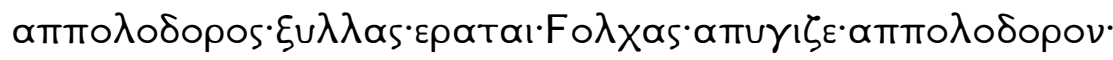

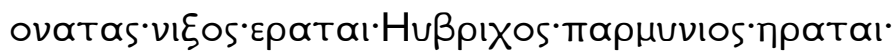

L'interprétation donnée par A. Pontrandolfo (Pontrandolfo, 1987: 58-59) restitue deux phrases: "Apollodore aime Ksyllas / Vulchas sodomise Apollodore » et "Onatas aime Niksos / Ybrichos a aimé Parmynios ». L'étude de l'onomastique montre tout d'abord la présence d'au moins cinq personnes de sexe masculin (Apollodore deux fois, Vulchas, Onatas, Ybrichos et Parmynios), tandis que Ksylla et Niksos doivent être des noms féminins. Ces noms de femmes ainsi que Ybrichos et Parmynios sont absents du répertoire onomastique grec, ce qui fait au total quatre noms propres sans une origine déterminée. Il s'agit probablement des noms italiques transcrits à la manière grecque. Apollodore, présent deux fois, et Onatas sont des noms grecs plus ou moins courants et Vulchas renvoie certainement à un nom étrusque.

En plus de l'origine ethnique des personnages, cette olpè indique des éléments culturels de large diffusion en Méditerranée : la consommation du vin, puisque cette forme céramique est associée au symposion grec, ainsi que la pratique de la pédérastie. L'olpè est un vase relativement marginal dans le service du banquet, mais l'ensemble du mobilier de la tombe, avec coupe et skyphos, renvoie sans aucun doute à la 
consommation du vin. De plus, la pratique de la pédérastie, clairement affichée par le texte de l'inscription, mène à l'identification du symposion de type grec plutôt qu'à la forme étrusque du banquet ${ }^{18}$.

Comme l'a remarqué A. Pontrandolfo, cette olpè inscrite est importante car elle atteste des rapports de réciprocité qui dépassent les simples échanges commerciaux, sur lesquels on est déjà suffisamment informé par ailleurs (Pontrandolfo, 1987: 62-63) ${ }^{19}$. En effet, les premières importations de matériel céramique grec en milieu étrusco-campanien datent du deuxième quart du VIIIe siècle et démontrent un commerce intense entre ces centres indigènes et les premières colonies grecques établies sur les côtes tyrrhéniennes 20 .

En effet, ce document exceptionnel témoigne de plusieurs marqueurs d'interaction culturelle. L'objet même de l'olpè est un vase d'importation de la cité grecque voisine de Poseidonia. Puis, l'ensemble du mobilier appartient au service du banquet, mais la mention de la pédérastie renvoie plutôt à la pratique du symposion de type grec qu'aux formes étrusques du banquet. De plus, l'étude onomastique identifie les personnes mentionnées comme étant à la fois des hommes et des femmes, d'origine grecque, étrusque et indigène. Enfin, l'objet lui-même témoigne de rapports complexes puisqu'il a été fabriqué et gravé dans un atelier de la cité grecque de Poseidonia, mais il a composé le mobilier d'une tombe de Fratte, cité étrusque de Campanie.

\section{Conclusion}

Les quatre cas étudiés, l'inscription de Tortora, le dossier numismatique des communautés sous l'influence de l' «empire » de Sybaris, l'ostrakon de Pisticci et l'olpè de Fratte, montrent différentes formes d'interaction culturelle et interethnique dans le contexte colonial d'Italie méridionale. Tous mettent en relief certains modes d'expression portés par les colons grecs et empruntés par des communautés italiques: tout d'abord l'utilisation de l'alphabet grec, mais aussi l'usage d'inscriptions, et a fortiori de bornes inscrites, de monnaies avec l'indication d'ethniques, des pratiques de la pédérastie et du banquet de type grec.

\footnotetext{
18 Sur une approche comparée des différentes formes de banquet et de consommation de vin dans la Méditerranée antique, voir en particulier Esposito, 2015.

${ }^{19}$ L'auteur fait également référence à une autre inscription grecque trouvée dans une tombe à Pontecagnano, qui porte le nom Dymeiada au génitif. La particularité de l'inscription de Fratte, par rapport à celle de Pontecagnano, est justement la possibilité de la lier à certaines pratiques culturelles dans un milieu indigène. Cet élément doit être mis en rapport, entre autres, avec les implications « ethniques » de l'iconographie de la tombe du Plongeur de Poseidonia.

20 Pour le commerce étrusque à l'époque archaïque, voir Gras, 1985, en particulier sur Pontecagnano voir p. 486-488.
} 
Ces objets et textes mis en contexte ont montré des nuances importantes dans l'interprétation des emprunts de ces formes d'expression. Tout d'abord, leur utilisation n'implique pas nécessairement l'acceptation des valeurs grecques. Inversement, les communautés italiques peuvent choisir, en ce qui concerne certaines pratiques comme le banquet, les formes plutôt grecques que celles utilisées majoritairement dans leur cadre culturel habituel, traduisant une adhésion plus complète des mœurs grecques. Mais le trait le plus remarquable est probablement l'utilisation des formes grecques dont la fonction était l'affirmation de l'autonomie politique de communautés italiques. Il s'agit ainsi d'un mouvement en quelque sorte dialectique, où les indigènes empruntent des moyens d'expression de type grec pour marquer leur autonomie, du moins relative, par rapport aux cités grecques.

En définitive, on voit que l'étude de documents selon l'approche de l'archéologie historique, où l'on confronte textes et culture matérielle sans hiérarchie entre les types de sources, offre la possibilité de saisir des messages ambivalents, où la forme et le contenu sont analysés en parallèle et peuvent montrer des interactions complexes entre populations d'origines diverses.

\section{Bibliographie}

ARENA, Renato. 1996. Iscrizioni greche arcaiche di Sicilia e Magna Grecia. IV : Iscrizioni delle colonie achee, Alessandria: Edizioni dell'Orso.

BARBERIS, Valentina. 1999. " I siti tra Sinni e Bradano dall'età arcaica all'età ellenistica: schede ", in BARRA BAGNASCO, Marcella, DE MirO, Ernesto e PINZONE, Antonino (orgs.), Magna Grecia e Sicilia. Stato degli studi e prospettive di ricerca, Magna Grecia e Sicilia. Stato degli studi e prospettive di ricerca:59-105, Messine: Di.Sc.A.M.

BERTRAND, Jean-Marie. 2004. Inscriptions historiques grecques. La Roue à livres / documents, Paris: Les Belles Lettres. Primeira edição, 1992.

BotTINI, Angelo e LeCCE, Lucia. 2013. " La mesogaia lucana e il caso di Pisticci. », in TODISCO, Luigi (org.), La comunicazione verbale tra Greci e indigeni in Apulia nel V-IV secolo a. C. : quali elementi?, La comunicazione verbale tra Greci e indigeni in Apulia nel V-IV secolo a. C. : quali elementi?:45-60, Naples: Loffredo.

BOURDIN, Stéphane. 2012. Les peuples de l'Italie préromaine : identités, territoires et relations inter-ethniques en Italie centrale et septentrionale (VIII ${ }^{e-I}{ }^{e r}$ s. av. J.-C.). Bibliothèque des Écoles Françaises d'Athènes et de Rome, 350, Rome: École française de Rome. 
BROUSSEAU, Louis. 2010. " Le monnayage des Serdaioi revisité ». Revue numismatique, 166, p. 257-285.

Bugno, Maurizio. 1999. Da Sibari a Turi. La fine di un impero. Études. III, Naples: Centre Jean Bérard.

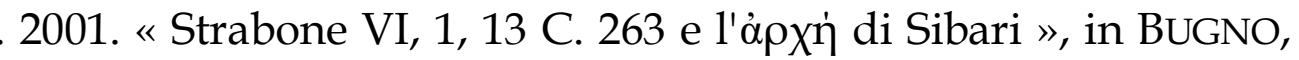
Maurizio e MASSERIA, Concetta (orgs.), Il mondo enotrio tra VI e $V$ secolo a.C., Il mondo enotrio tra VI e V secolo a.C.:303-327, Naples: Loffredo.

CASTOLDI, Marina. 2007. " Nuove indagini archeologiche nel Metapontino, tra Pisticci e Ferrandina ». Acme, Anali della fcoltà di lettere e filosofia dell'Università di Milano, 60, 1, p. 249-260.

CUNLIFFE, Barry. 2006. " Afterword: historical archaeology in the wider discipline ", in HICKS, Dan e BEAUDRY, Mary C. (orgs.), The Cambridge companion to Historical archaeology, The Cambridge companion to Historical archaeology:314-319, Cambridge: Cambridge University Press.

DE JULIIS, Ettore Maria. 1996. Magna Grecia. L'Italia meridionale dalle origini leggendarie alla conquista romana. Temi e luoghi del mondo antico. 2, Bari: Edipuglia.

DenOyelLe, Martine. 1997. "Attic or non-Attic? The case of the Pisticci Painter ", in OAKLEY, John Howard, COULSON, William D. E. e PALAGIA, Olga (orgs.), Athenian potters and painters : the conference proceedings, Athenian potters and painters : the conference proceedings:395-405, Oxford: Oxbow books.

DeNOYelLE, Martine e IOZZO, Mario. 2009. La céramique grecque d'Italie méridionale et de Sicile : productions coloniales et apparentées du VIIIe au IIIe siècle av. J.-C. Les Manuels d'art et d'archéologie antiques. La céramique grecque, 4, Paris: Picard.

DuBOIS, Laurent. 2002. Inscriptions grecques dialectales de Grande Grèce. Tome II, Colonies achéennes. Hautes études du monde gréco-romain, 30, Genève: Droz.

DYSON, Stephen. 1995. "Is there a text in this site? ", in SMALL, David B. (org.), Methods in the Mediterranean: historical and archaeological views on texts and archaeology, Methods in the Mediterranean: historical and archaeological views on texts and archaeology:25-44, Leiden: Brill. 
ESPOSITO, Arianna (org.) 2015. Autour du "banquet" : modèles de consommation et usages sociaux, Sociétés, Dijon: Éditions universitaires de Dijon.

FUnARI, Pedro Paulo Abreu. 1999. " Historical archaeology from a world perspective », in FUNARI, Pedro Paulo Abreu, HALL, Martin e JONES, Siân (orgs.), Historical Archaeology: back from the edge, Historical Archaeology: back from the edge:37-66, Londres: Routledge.

FunARI, Pedro Paulo Abreu, Oliveira, Nanci, e ZARANKIN, Andrés (orgs.). 2010. Contemporary issues in historical archaeology, Proceedings of the XV world congress, Lisbon, 4-9 september 2006, Oxford: Archaeopress.

FUnARI, Pedro Paulo Abreu, ZARANKIN, Andrés, e STOVEL, Emily (orgs.). 2005. Global archaeological theory: contextual voices and contemporary thoughts, New York: Kluwer academic/Plenum publishers.

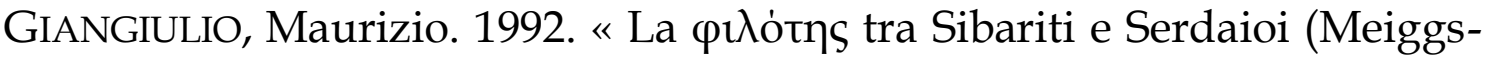
Lewis, 10) ». Zeitschrift für Papyrologie und Epigraphik, 93, p. 31-44.

GRAS, Michel. 1985. Trafics tyrréniens archaïques. Bibliothèques des écoles françaises d'Athènes et de Rome. 258, Rome: École Française de Rome.

GRECO, Emanuele. 1990. "Serdaioi ». Annali dell'Istituto Universitario Orientale di Napoli, Sezioni di Archeologia e storia antica, p. 39-57.

.1992a. Archeologia della Magna Grecia, Rome-Bari: Laterza.

1992b. «L'impero di Sibari: bilancio archeologico-topografico». in Sibari e la Sibaritide. Atti del convegno di studi sulla Magna Grecia. XXXII:459-485, Tarente: Istituto per la Storia e l'Archeologia della Magna Grecia.

2013. "Sul cosiddetto 'impero' di Sibari fino alla tirannide di Telys ed alla distruzione della città », in DELIA, GIORGIO e MASNERI, TULLIO (orgs.), Sibari : archeologia, storia, metafora, Sibari : archeologia, storia, metafora:197-203, Castrovillari: Il coscile.

GRECO, Emanuele e GASPARRI, Domenico (orgs.). 1995. Laos, Città e territorio nelle colonie greche d'Occidente, 2, Tarente: Istituto per la Storia e l'Archeologia della Magna Grecia.

GRECO, Emanuele, LONGO, Fausto, DI CESARE, Riccardo, e MARCHIANDI, Daniela (orgs.). 2014. Topografia di Atene : sviluppo urbano e monumenti dalle origini al III secolo d.C. Tomo 3, Quartieri a nord e a nord-est dell'Acropoli e 
Agora del Ceramico, GRECO, Emanuele (org.), Studi di archeologia e di topografia di Atene e dell'Attica (SATAA), 1.3, 2 vols., Athènes: Scuola Archeologica Italiana di Atene.

GRECO, Giovanna e PONTRANDOlFO, Angela (orgs.). 1990. Fratte un insediamento etrusco-campano, Modena: Franco Cosimo Panini.

HANSEN, Mogens Herman. 1976. Apagoge, endeixis and ephegesis against kakourgoi, atimoi and pheugontes : a study in the athenian administration of justice in the fourth century. Odense University classical studies, 8, Odense: Odense University Press.

HORSNAES, Helle W. 2011. "Coinages of indigenous communities in archaic Southern Italy - the mint as a means of promoting identity? », in GLEBA, Margarita e HORSNAES, Helle W. (orgs.), Communicating identity in Italic Iron age communities, Communicating identity in Italic Iron age communities:197-209, Oxford: Oxbow books.

JOHNSON, Matthew H. 1999. "Rethinking historical archaeology ", in FUNARI, Pedro Paulo Abreu, HALL, Martin e JONES, Siân (orgs.), Historical Archaeology: back from the edge, Historical Archaeology: back from the edge:23-36, Londres: Routledge.

LA TORRE, Gioacchino Francesco. 1995. «L'iscrizione di San Brancato », in LA TORRE, Gioacchino Francesco (org.), Nuove testimonianze di archeologia calabrese : greci, indigeni e romani nell'Alto Tirreno Cosentino, Nuove testimonianze di archeologia calabrese : greci, indigeni e romani nell'Alto Tirreno Cosentino:29-31, Rome: Stampa Recografica.

LALONDE, Gerald V., LANGDON, Merle K., e WaLbanK, Michael B. 1991. Inscriptions : horoi, poletai records, leases of public lands. The Athenian agora, 19, Princeton: American School of Classical Studies at Athens.

LAWRENCE, Susan e SHEPHERD, Nick. 2006. «Historical archaeology and colonialism », in HICKS, Dan e BEAUDRY, Mary C. (orgs.), The Cambridge companion to historical archaeology, The Cambridge companion to historical archaeology:69-86, Cambridge: Cambridge University Press.

LAZZARINI, Maria Letizia e POCCETTI, Paolo. 2001. Il mondo enotrio tra VI e $V$ secolo a.C. L'iscrizione paleoitalica da Tortora. Atti dei seminari napoletani, 1996-1998, Quaderni di ostraka, 1, 2, Naples: Loffredo.

LOMBARDO, Mario. 2008. « Il trattato tra i Sibariti e i Serdaioi: problemi di cronologia e di inquadramento storico », in DE SENSI SESTITO, Giovanna (org.), La Calabria tirrenica nell'antichità : nuovi documenti e problematiche 
storiche, La Calabria tirrenica nell'antichità : nuovi documenti e problematiche storiche:219-232, Soveria Mannelli: Rubbettino.

MESKELL, Lynn. 2001. « Archaeologies of identity », in HODDER, Ian (org.), Archaeological theory today, Archaeological theory today:187-213, Cambridge: Polity.

Nouveau choix d'inscriptions grecques : textes, traductions et commentaires par l'Institut Fernand-Courby. 2005. Paris: Les Belles Lettres.

OBER, Josiah. 1995. «Greek Horoi: artifactual texts and the contingency of meaning ", in SMALL, David B (org.), Methods in the Mediterranean: historical and archaeological views on texts and archaeology, Methods in the Mediterranean: historical and archaeological views on texts and archaeology:91-123, Leiden: Brill.

ORSER JR, Charles E. 2000. Introducción a la arqueología histórica. Tradução de ZARANKIN, Andrés, Buenos Aires: Asociación amigos del Instituto nacional de antropología. Primeira edição, 1992.

(org.) 2002. Encyclopaedia of Historical Archaeology, Londres: Routledge.

OSANNA, Massimo. 1992. Chorai coloniali da Taranto a Locri : documentazione archeologica e ricostruzione storica, Rome: Istituto Poligrafico e Zecca dello Stato.

PARISE, Nicola Franco. 2001. «Intorno alle serie minori d'incusi di AMI, di PAL-MOL e di SO », in BUGNO, Maurizio e MASSERIA, Concetta (orgs.), Il mondo enotrio tra VI e V secolo a.C. , Il mondo enotrio tra VI e V secolo a.C. :139-143, Naples: Loffredo.

POLOSA, Annalisa. 2000. «Vecchie e nuove ipotesi sui Serdaioi: una messa a punto ». Annali di archeologia e storia antica, n.s. 7, p. 49-59.

PONTRANDOLFO, Angela. 1987. «Un'iscrizione posidoniate in una tomba di Fratte di Salerno ». Annali dell'Istituto Universitario Orientale di Napoli, Sezioni di Archeologia e storia antica, IX, p. 55-63.

SCOTT, Joan W. 1986. " Gender: a useful category of historical analysis ». The American Historical Review, vol. 91, 5, p. 1053-1075.

SHEAR, Thedore Leslie. 1939. « The campaign of 1938 ». Hesperia, 8, p. 201246. 1940. « The campaign of $1939 »$. Hesperia, 9, p. 261-308. 
SMALL, David B. 1995a. "Introduction ", in SMALL, David B. (org.), Methods in the Mediterranean: historical and archaeological views on texts and archaeology, Methods in the Mediterranean: historical and archaeological views on texts and archaeology:1-22, Leiden: Brill.

1995b. « Monuments, laws, and analysis: combining archaeology and text in Ancient Athens ", in SMALL, David B. (org.), Methods in the Mediterranean: historical and archaeological views on texts and archaeology, Methods in the Mediterranean: historical and archaeological views on texts and archaeology:143-174, Leiden: Brill.

. 1999. "The tyranny of the text: lost social strategies in current historical period archaeology in the classical Mediterranean », in FUNARI, Pedro Paulo Abreu, HALl, Martin e JONES, Siân (orgs.), Historical Archaeology: back from the edge, Historical Archaeology: back from the edge:122-136, Londres: Routledge.

TAGLIENTE, Marcello e LOMBARDO, Mario. 1985. "Nuovi documenti su Pisticci in età arcaica. 1. Lo scavo, 2. Il graffito ». La parola del passato, 223, p. 284-307.

TALIERCIO MENSITIERI, Marina. 2001. « La monetazione degli Enotri », in BUGNO, Maurizio e MASSERIA, Concetta (orgs.), Il mondo enotrio tra VI e V secolo a.C., Il mondo enotrio tra VI e V secolo a.C.:117-137, Naples: Loffredo.

THOMPSON, Homer A. 1968. " Activity in the Athenian Agora: 1966-1967 ». Hesperia. The Journal of the American School of Classical Studies at Athens, 37,1 , p. 36-72.

VAN EFFENTERRE, Henri e RUZE, Françoise. 1994. Nomima : recueil d'inscriptions politiques et juridiques de l'archaïsme grec. Vol. I, Cités et institutions. Collection de l'École française de Rome, 188, Rome: École française de Rome. 